\title{
Impact of bioprosthetic valve type on peri-procedural myocardial injury and mortality after transcatheter aortic valve replacement
}

\author{
Vincenzo De Marzo ${ }^{1,2}$. Gabriele Crimi ${ }^{1}$. Matteo Vercellino ${ }^{1}$. Stefano Benenati ${ }^{1,2}$. Fabio Pescetelli ${ }^{1,2}$. \\ Roberta Della Bona ${ }^{1} \cdot$ Matteo Sarocchi ${ }^{1,2} \cdot$ Marco Canepa $^{1,2} \cdot$ Manrico Balbi $^{1,2} \cdot$ Italo Porto $^{1,2}$ (])
}

Received: 5 February 2021 / Accepted: 23 April 2021 / Published online: 7 May 2021

(c) The Author(s) 2021

\begin{abstract}
Peri-procedural myocardial injury (PPMI) is a common complication after transcatheter valve replacement (TAVR), often remaining clinically silent. The role of valve type on PPMI and the association between PPMI and mortality are still unclear. We sought to evaluate predictors and outcome of PPMI after TAVR, and the impact of self-expandable valve (SEV) vs. balloon-expandable valve (BEV) deployment on PPMI. Consecutive patients who underwent successful TAVR in a singlecenter from January 2014 to December 2019 were included. PPMI was defined according to a modified Valve Academic Research Consortium (VARC)-2 definition as a post-procedure elevation of troponin (with a peak value $\geq 15$-times the upperreference limit $)<72 \mathrm{~h}$ after TAVR. We included 596 patients, of whom $258(43.3 \%)$ were men. Mean age was $83.4 \pm 5.5$ years. We deployed $368(61.7 \%)$ BEV and 228 (38.3\%) SEV. PPMI was observed in 471 (79.0\%) patients. At multivariable analysis, SEV (OR 2.70, 95\% CI 1.64-4.55, $p<0.001$ ), creatinine clearance (OR 0.98, 95\% CI 0.97-1.00, $p=0.011$ ), and baseline ejection fraction (OR 1.05, 95\% CI 1.02-1.07, $p<0.001$ ) were independent predictors of PPMI; these findings were also confirmed using a propensity-weighted analysis. Thirty-day and 1-year all-cause mortality rates were $2.5 \%$ and $8.1 \%$, respectively. No associations between PPMI and 30-day $(p=0.488)$ or 1-year $(p=0.139)$ all-cause mortality were found. Independent predictors of 30-day mortality were increasing EUROSCORE II (HR 1.16 per score point, 95\% CI 1.08-1.19, $p<0.001$ ) and life-threatening/major bleeding complications (HR 5.87, 95\% CI 1.28-26.58, $p=0.019$ ), whereas EUROSCORE II (HR 1.08, 95\% CI 1.04-1.13, $p=0.031$ ) and acute kidney injury (HR 2.59, 95\% CI 1.20-5.35, $p=0.020$ ) were related to 1-year mortality. PPMI is frequent after TAVR, but it does not affect 30-day or 1-year all-cause mortality. SEV implantation is associated with an increased frequency of PPMI.
\end{abstract}

Keywords Transcatheter aortic valve replacement $\cdot$ Heart valve prosthesis $\cdot$ Troponin $\cdot$ Heart injuries $\cdot$ Creatine kinase MB form · Troponin I

\section{Introduction}

Transcatheter aortic valve replacement (TAVR) is the treatment of choice for severe aortic stenosis (AS) at high risk for conventional surgery, and it is increasingly being performed in patients at intermediate and low risk [1-4]. As the TAVR

Italo Porto

italo.porto@unige.it

1 DICATOV-Cardiothoracic and Vascular Department, San Martino Hospital, IRCCS for Oncology and Neurosciences, Genoa, Italy

2 Department of Internal Medicine and Specialties (DIMI), Clinic of Cardiovascular Diseases, University of Genoa, Viale Benedetto XV, 10, 16132 Genoa, Italy community moves towards treating less complex patients, recognition and management of even minor intra- and periprocedural complications becomes pivotal.

Among complications of TAVR, peri-procedural myocardial injury (PPMI) is common [1-4], occurring in roughly half of procedures if a sensitive definition is used [1, 5-13]. The etiopathogenesis of PPMI after TAVR (PPMI-TAVR) is multifactorial, depending on both patient-related factors, such as aortic atheroma burden, as well as procedural factors, such as pre-TAVR balloon valvuloplasty, rapid pacing, and valve post-dilatation $[1,8,10-13]$. In this regard, there might be an increased risk of PPMI-TAVR if a self-expandable valve (SEV), as compared to a balloon-expandable valve (BEV), is chosen. Indeed, the profoundly different valve designs and implantation techniques may affect procedural 
time, and the tissue compression by valve frames is clearly different.

The impact of PPMI on prognosis, however, remains controversial [2, 14-17], as most data derive from observational studies applying different PPMI definitions to heterogeneous populations.

Therefore, the primary aim of our retrospective study was to determine the incidence, predictors and impact of SEV (as compared to BEV) deployment on PPMI; the secondary aim was to explore the effect of PPMI (and of SEV vs. BEV) on clinical outcomes.

\section{Materials and methods}

\section{Study population}

This is a retrospective study using a prospectively maintained database. All consecutive patients with symptomatic severe AS who had undergone TAVR between January 2014 and December 2019 at our institution were included. Therefore, we excluded peri-procedural deaths and patients with incorrect positioning of valve for improper anatomical location or use of $>1$ valve.

All patients signed an informed consent allowing the utilization of their anonymized clinical information for medical research purposes, as approved by the local Institutional Review Board (Genova TAVR registry, $\mathrm{N}^{\circ}$ Registro CER Liguria: 331/2020—DB id 10,646).

\section{Demographic and clinical data}

For every patient, we collected gender, age, body mass index (BMI), New York Heart Association (NYHA) class, diabetes mellitus (DM), chronic kidney disease (CKD), chronic obstructive pulmonary disease (COPD), history of neurological disease, coronary artery disease (CAD), previous acute coronary syndrome (irrespective of myocardial revascularization), atrial fibrillation (AF), prior valvuloplasty, and European System for Cardiac Operative Risk Evaluation score II (EUROSCORE II).

$\mathrm{CAD}$ was defined as the presence of at least one coronary stenosis $\geq 50 \%$ in vessels $\geq 1.5 \mathrm{~mm}$. Echocardiography data, e.g., left ventricular ejection fraction (LVEF), maximum and mean aortic valve gradient were also collected.

\section{Laboratory values and measurements}

Among biomarkers, creatinine, creatinine clearance $(\mathrm{CrCl})$ estimated by Cockcroft-Gault formula, and troponin I ( TnI) were assessed daily until hospital discharge.

Plasma TnI concentration was measured using a sandwich chemiluminescent immunoassay based on $\mathrm{LOCI}^{\circledR}$ technology on Dimension Vista ${ }^{\circledR} 1500$ System. The limit of quantitation (functional sensitivity), which corresponds to the TnI concentration at which the coefficient of variation is $10 \%$, was $<0.04 \mu \mathrm{g} / \mathrm{L}$ [18]. The upper-reference limit (URL), as defined at the 99th percentile of the reference interval, was $0.046 \mu \mathrm{g} / \mathrm{L}$.

\section{Procedural data}

TAVR-related data such as procedural time, type of vascular access, type of valve, valve-in-valve implantation, preand post-TAVR dilatation, and the use of rapid pacing were recorded.

Successful TAVR were defined according to the presence of all the following criteria: absence of procedural mortality; correct positioning of a single prosthetic heart valve into the proper anatomical location; intended performance of the prosthetic heart valve (no prosthesis-patient mismatch and mean aortic valve gradient $<20 \mathrm{mmHg}$ or peak velocity $<3$ $\mathrm{m} / \mathrm{s})[19]$.

For the purpose of this analysis, we included the following SEV: CoreValve (Medtronic, Minneapolis, Minnesota), Evolut R (Medtronic, Minneapolis, Minnesota), Evolut PRO (Medtronic, Minneapolis, Minnesota), Symetis ACURAT E neo (Symetis/Boston, Ecublens, Switzerland) and NVT ALLEGRA (New Valve Technology [NVT], Hechingen, Germany). SAPIEN XT (Edwards Lifesciences, Irvine, California), SAPIEN 3 (Edwards Lifesciences, Irvine, California), and SAPIEN 3 ULTRA (Edwards Lifesciences, Irvine, California) were considered BEV.

\section{VARC-2 definitions}

Length of hospital stay, VARC-2 outcomes (PPMI, AKI, stroke, vascular complications, bleedings, permanent pacemaker implantation, cardiac tamponade), and mortality at 30-day and 1-year follow-up were collected.

PPMI was defined according to a modified Valve Academic Research Consortium (VARC)-2 criteria as a postprocedure elevation of troponin (with a peak value exceeding $15 \times$ as the upper-reference limit) within $72 \mathrm{~h}$ after TAVR, at least in one sample. If troponin was increased at baseline ( $>99$ th percentile), a further post-procedural increase of at least $50 \%$ was required to meet the endpoint definition [19]. Since ischemic symptoms in the peri-procedural setting appear misleading and confounding in nature, we decided to focus only on the laboratory markers (e.g., troponin elevation) to define PPMI.

AKI was defined as an increase of at least $0.3 \mathrm{mg} / \mathrm{dL}$ in serum creatinine or a urine output worsening $(<0.5 \mathrm{~mL} / \mathrm{kg}$ for $<12 \mathrm{~h}$ ) occurring within 7 days after the procedure [19]. 


\section{Statistics}

Categorical variables were expressed as frequencies and percentages and compared by Chi-square test or Fisher's exact-test; continuous variables were reported as mean and relative standard deviation (SD) or median and interquartile range (IQR) and were compared using the unpaired Student $t$ test or the Wilcoxon rank-sum test, depending on the variable distribution.

Univariate logistic regression analysis was used to identify clinical and procedural factors associated with PPMI. Thereafter, a multivariate logistic regression model was fitted, including all variables with $p$ values $<0.10$ in the univariate analysis, plus age and sex as background variables, to explore the independent impact of those variables on the development of PPMI.

Univariate survival analysis was performed fitting Kaplan-Meier curves (time to all-cause death at 30-day and 1-year) for patients developing or not PPMI. Subsequently, uni- and multivariate Cox models (for 30-day mortality and 1-year mortality) were constructed to estimate the hazard ratios (HRs) and 95\% confidence interval (CI) of baseline, procedural characteristics, and VARC-2 outcomes on 30-day and 1-year mortality. The variables were selected on a statistical and clinical basis. In particular, laboratory and procedural variables with a $p<0.10$ association with the outcome of interest were included (avoiding collinearity), and clinically meaningful predictors were also assessed based on an updated literature search [19].

Sensitivity analysis using multiple imputation with predictive mean matching and the exclusion of the covariates with missingness were used.

Finally, to better investigate the role of type of vascular access, we repeated the statistical analysis for PPMI and 30-day/1-year mortality excluding those patients who received transapical access due to its higher invasiveness.

A two-tailed $p$ value of 0.05 was considered to reject the null hypothesis. All analyses were performed with $\mathrm{R}$ environment 3.6.3 (R Foundation for Statistical Computing, Vienna, Austria) using tableone, finalfit, survival, and twang packages [20].

\section{Results}

We included 695 patients who underwent TAVR at our institution from January 2014 to December 2019. Of those, 23 (3.3\%) patients were excluded due to unsuccessful TAVR (11 for peri-procedural deaths, 12 for incorrect positioning of valve for improper anatomical location or use of $>1$ valve) and $76(10.9 \%)$ due to the lack of appropriate biomarkers measurements before or during hospital stay, leading to a final study cohort of 596 patients. Baseline clinical features are outlined in Table 1. Mean age was $83.4 \pm 5.5$ years and $258(43.3 \%)$ of patients were men, median EUROSCORE II was $4.1 \%$ (IQR 3.3-6.5). Two-hundred sixty-seven (44.8\%) patients had CAD, baseline median creatinine was 1.1 (IQR $0.9-1.4) \mathrm{mg} / \mathrm{dL}$, whereas baseline median $\mathrm{CrCl}$ was 37.5 (IQR 28.6-49.6) $\mathrm{mL} / \mathrm{min}$.

Median procedural time was 113 (IQR 86.0-146.0) min, $551(92.4 \%)$ procedures were transfemoral, $336(56.4 \%)$ patients underwent pre-dilatation, 57 (9.6\%) post-dilatation, whereas rapid pacing was used in $505(84.7 \%)$. Other procedural variables are shown in Table 2.

We implanted 29 (4.9\%) SAPIEN XT, 285 (47.8\%) SAPIEN 3, 54 (9.1\%) SAPIEN 3 ULTRA, and $5(0.8 \%)$ NVT ALLEGRA, 70 (11.7\%) CoreValve, 58 (9.7\%) Evolut R, 85 (14.3\%) Evolut PRO, and 10 (1.7\%) ACURATE neo.

\section{PPMI predictors}

Incidence of VARC-2 adverse events is presented in Table 3; PPMI was observed in 475 (79.0\%) patients.

Patients who developed PPMI was less frequent male (40.3\% vs $54.4 \%, p=0.007$ ) and had less history of CAD ( $41.8 \%$ vs. $56.0 \%, p=0.006)$ or diabetes $(25.3 \%$ vs. $37.6 \%$, $p=0.012$ ); furthermore, patients with PPMI had higher baseline LVEF (55.0 [IQR 50.0-60.0] vs. 55.0 [IQR 40.0-55.0], $p=0.001$ ), lower baseline $\mathrm{CrCl}$ (36.8 [IQR $27.6-48.1$ ] $\mathrm{mL} / \mathrm{min}$ vs. 41.3 [IQR 32.0-53.8], $p=0.001$ ), and were implanted more frequent with SEV rather than BEV ( $42.5 \%$ vs. $22.4 \%, p<0.001)$.

After adjusting for confounders in the multivariable analysis, we found that SEV implantation (OR 2.70, 95\% CI 1.64-4.55, $p<0.001$ ), lower baseline $\mathrm{CrCl}$ (OR 0.98, 95\% CI 0.97-1.00, $p=0.011$ ), and higher LVEF (OR 1.05, 95\% CI 1.02-1.07, $p<0.001)$ were independent predictors of PPMI (Table 4). Notably, SEV, baseline $\mathrm{CrCl}$, and LVEF remained independently associated with higher PPMI even when pre-dilation, post-dilatation, and the use of rapid pacing were forced into the model to account for procedural potential confounders (Table 5).

Furthermore, after well balancing SEV vs. BEV with an average treatment effect propensity score (Fig. 1), we confirmed a significant higher incidence of PPMI among patients receiving SEV as compared to $\mathrm{BEV}(p<0.001)$.

Finally, SEV implantation, baseline $\mathrm{CrCl}$, and LVEF remained significant when we repeated the regression analysis for PPMI excluding those patients who received transapical access (Table 6).

\section{All-cause mortality}

Overall, at a median follow-up of 18.5 (IQR 7.6-34.0) months, we observed 15 (2.5\%) 30-day and 48 (8.1\%) 1 -year all-cause deaths. PPMI was not associated with 
Table 1 Baseline patient's characteristics. All measures expressed as $n(\%)$, mean (SD) or median with interquartile range (quartile 1 to quartile 3)

\begin{tabular}{|c|c|c|c|c|}
\hline Variable & $\begin{array}{l}\text { Overall } \\
(n=596)\end{array}$ & $\begin{array}{l}\text { No PPMI } \\
(n=125)\end{array}$ & $\begin{array}{l}\text { PPMI } \\
(n=471)\end{array}$ & $p$ value \\
\hline Age & $83.4(5.5)$ & $83.1(5.7)$ & $83.5(5.4)$ & 0.420 \\
\hline Men & $258(43.3)$ & $68(54.4)$ & $190(40.3)$ & 0.007 \\
\hline BMI & $25.3(4.2)$ & $25.6(4.4)$ & $25.2(4.1)$ & 0.274 \\
\hline \multicolumn{5}{|l|}{ NYHA } \\
\hline Class I & $31(5.2)$ & $7(5.6)$ & $24(5.1)$ & 0.012 \\
\hline Class II & $246(41.3)$ & $44(35.2)$ & $202(42.9)$ & \\
\hline Class III & $280(47.0)$ & $58(46.4)$ & $222(47.1)$ & \\
\hline Class IV & $39(6.5)$ & $16(12.8)$ & $23(4.9)$ & \\
\hline EUROSCORE II & $4.4(3.3-6.5)$ & $4.6(3.4-7.0)$ & $4.3(3.2-6.4)$ & 0.194 \\
\hline Diabetes & $166(27.9)$ & $47(37.6)$ & $119(25.3)$ & 0.012 \\
\hline Previous acute coronary syndrome & $108(18.1)$ & $29(23.2)$ & $79(16.8)$ & 0.129 \\
\hline CAD & $267(44.8)$ & $70(56.0)$ & $197(41.8)$ & 0.006 \\
\hline Previous valvuloplasty & $52(8.7)$ & $16(12.8)$ & $36(7.6)$ & 0.097 \\
\hline Pre-TAVR creatinine (mg/dL) & $1.1(0.9-1.4)$ & $1.1(0.9-1.4)$ & $1.1(0.9-1.4)$ & 0.914 \\
\hline Pre-TAVR CrCl (mL/min) & $37.5(28.6-49.6)$ & $41.3(32.0-53.8)$ & $36.8(27.6-48.1)$ & 0.012 \\
\hline Baseline hemoglobin (mg/dL) & $12.0(10.9-13.1)$ & $12.2(11.5-13.2)$ & $11.9(10.8-12.9)$ & 0.015 \\
\hline Baseline NT-proBNP (ng/dL) & $3401.0(1564.8-7788.3)$ & $3897.0(2023.0-8023.0)$ & $3258.0(1403.5-7672.5)$ & 0.252 \\
\hline Atrial fibrillation & $205(34.4)$ & $47(37.6)$ & $158(33.5)$ & 0.466 \\
\hline COPD & $141(23.7)$ & $36(28.8)$ & $105(22.3)$ & 0.160 \\
\hline LVEF $(\%)$ & $55.0(48.0-60.0)$ & $55.0(40.0-55.0)$ & $55.0(50.0-60.0)$ & $<0.001$ \\
\hline Maximum aortic gradient (mmHg) & $100.0(82.0-112.0)$ & $96.0(69.8-108.3)$ & $99.0(80.0-112.0)$ & 0.052 \\
\hline Mean aortic gradient $(\mathrm{mmHg})$ & $51.5(44.0-60.0)$ & $49.0(40.0-60.0)$ & $51.5(44.8$ to 60.0$)$ & 0.056 \\
\hline
\end{tabular}

$B M I$ body mass index, $B N P$ brain natriuretic peptide, $C A D$ coronary artery disease, $C O P D$ chronic obstructive pulmonary disease, $C r C l$ creatinine clearance, $L V E F$ left ventricular ejection fraction, $N Y H A$; new york heart association, $P P M I$ post-procedure myocardial injury, TAVR transcatheter aortic valve replacement

Table 2 Procedural characteristics. All measures expressed as $\mathrm{n}(\%)$, mean $(\mathrm{SD})$ or median with interquartile range (quartile 1 to quartile 3)

\begin{tabular}{|c|c|c|c|c|}
\hline Variable & $\begin{array}{l}\text { Overall } \\
(n=596)\end{array}$ & $\begin{array}{l}\text { No PPMI } \\
(n=125)\end{array}$ & $\begin{array}{l}\text { PPMI } \\
(n=471)\end{array}$ & $p$ value \\
\hline Valve type & & & & $<0.001$ \\
\hline SAPIEN XT & $29(4.9)$ & $9(7.2)$ & $20(4.2)$ & \\
\hline SAPIEN 3 & $285(47.8)$ & $71(56.8)$ & $214(45.4)$ & \\
\hline SAPIEN 3 ULTRA & $54(9.1)$ & $17(13.6)$ & $37(7.9)$ & \\
\hline CoreValve & $70(11.7)$ & $6(4.8)$ & $64(13.6)$ & \\
\hline Evolut R & $58(9.7)$ & $5(4.0)$ & $53(11.3)$ & \\
\hline Evolut PRO & $85(14.3)$ & $15(12.0)$ & $70(14.9)$ & \\
\hline ACURATE neo & $10(1.7)$ & $1(0.8$ & $9(1.9)$ & \\
\hline NVT ALLEGRA & $5(0.8)$ & $1(0.8)$ & $4(0.8)$ & \\
\hline \multicolumn{5}{|l|}{ Vascular access } \\
\hline Femoral & $551(92.4)$ & $120(96.0)$ & $436(92.6)$ & 0.407 \\
\hline Transapical & $15(2.5)$ & $2(1.6)$ & $13(2.8)$ & \\
\hline Subclavian & $30(5.0)$ & $5(4.0)$ & $25(5.3)$ & \\
\hline Valve-in-valve & $18(3.0)$ & $5(4.0)$ & $13(2.8)$ & 0.670 \\
\hline Pre-dilatation & $336(56.4)$ & $64(51.2)$ & $272(57.7)$ & 0.341 \\
\hline Post-dilatation & $57(9.6)$ & $11(8.8)$ & $46(9.8)$ & 0.944 \\
\hline Rapid pacing & $505(84.7)$ & $111(88.8)$ & $394(83.7)$ & 0.301 \\
\hline Procedural time & 113.0 (86.0 to 146.0$)$ & $117.0(87.0$ to 140.8$)$ & $111.00(86.0$ to 147.0$)$ & 0.760 \\
\hline
\end{tabular}

$P P M I$ post-procedure myocardial injury 
Table 3 VARC-2 outcomes. All measures expressed as $n$ $(\%)$, mean (SD) or median with interquartile range (quartile 1 to quartile 3)

\begin{tabular}{llllr}
\hline Variable & $\begin{array}{l}\text { Overall } \\
(n=596)\end{array}$ & $\begin{array}{l}\text { No PPMI } \\
(n=125)\end{array}$ & $\begin{array}{l}\text { PPMI } \\
(n=471)\end{array}$ & $p$ value \\
\hline All-cause 30-day mortality & $15(2.5)$ & $2(1.6)$ & $13(2.8)$ & 0.679 \\
All-cause 1-year mortality & $48(8.1)$ & $6(4.8)$ & $42(8.9)$ & 0.167 \\
Cerebrovascular complication & $15(2.5)$ & $3(2.4)$ & $12(2.5)$ & 1.000 \\
Major stroke & $4(0.7)$ & $1(0.8)$ & $3(0.6)$ & \\
Minor stroke & $11(1.8)$ & $2(1.6)$ & $9(1.9)$ & $2.0(1.2-3.3)$ \\
Post-TAVR TnI & $1.5(0.8-2.8)$ & $0.4(0.2-0.6)$ & $85(18.0)$ & 0.001 \\
Bleeding complication & $99(16.6)$ & $14(11.2)$ & $8(1.7)$ & \\
Life-threatening & $9(1.5)$ & $1(0.8)$ & $24(5.1)$ & $53(11.3)$ \\
Major & $28(4.7)$ & $4(3.2)$ & $78(16.6)$ & 0.164 \\
Minor & $62(10.4)$ & $9(7.2)$ & $29(6.2)$ & \\
Vascular complication & $90(15.1)$ & $13(10.4)$ & $49(10.4)$ & 0.093 \\
Major & $32(5.4)$ & $3(2.4)$ & $1.2(0.9-1.6)$ & $<0.001$ \\
Minor & $59(9.9)$ & $10(8.0)$ & $34.1(24.1-46.4)$ & 0.214 \\
Post-TAVR creatinine (mg/dL) & $1.2(0.9-1.6)$ & $1.2(1.0-1.5)$ & $126(26.8)$ & 0.040 \\
Post-TAVR CrCl (ml/minute) & $35.3(25.6-47.4)$ & $40.6(31.7-54.0)$ & $74(15.7)$ & 0.976 \\
Acute kidney injury & $152(25.5)$ & $26(20.8)$ & $5(1.1)$ & \\
Permanent PM implantation & $84(14.1)$ & $10(8.0)$ & $2(1.6)$ & \\
Cardiac tamponade & $7(1.2)$ & &
\end{tabular}

$\mathrm{CrCl}$ creatinine clearance; $P M$ : pacemaker; $P P M I$ : post-procedure myocardial injury; TAVR: transcatheter aortic valve replacement; $T n I$ troponin I

\begin{tabular}{|c|c|c|c|c|c|c|}
\hline \multirow[t]{2}{*}{ Predictor } & \multicolumn{3}{|c|}{ Univariate } & \multicolumn{3}{|c|}{ Multivariate } \\
\hline & OR & $95 \% \mathrm{CI}$ & $p$ value & OR & $95 \% \mathrm{CI}$ & $p$ value \\
\hline Sex (male) & 0.56 & $0.37-0.82$ & 0.390 & 0.81 & $0.50-1.31$ & 0.390 \\
\hline Age & 1.01 & $0.98-1.05$ & 0.466 & 0.96 & $0.92-1.01$ & 0.111 \\
\hline SEV & 2.52 & $1.62-4.05$ & $<0.001$ & 2.70 & $1.64-4.55$ & $<0.001$ \\
\hline Pre-TAVR $\mathrm{CrCl}$ & 0.99 & $0.98-1.00$ & 0.031 & 0.98 & $0.97-1.00$ & 0.011 \\
\hline LVEF & 1.03 & $1.01-1.05$ & $<0.001$ & 1.05 & $1.02-1.07$ & $<0.001$ \\
\hline Diabetes & 0.57 & $0.38-0.87$ & 0.009 & 0.82 & $0.51-1.36$ & 0.443 \\
\hline CAD & 0.58 & $0.39-0.86$ & 0.007 & 0.67 & $0.42-1.07$ & 0.090 \\
\hline Maximum aortic gradient & 1.01 & $1.00-1.02$ & 0.014 & 1.01 & $1.00-1.02$ & 0.165 \\
\hline
\end{tabular}

$C A D$ coronary artery disease, $C r C l$ creatinine clearance, $L V E F$ left ventricular ejection fraction, $S E V$ selfexpandable valve, TAVR transcatheter aortic valve replacement
Table 4 Univariate and multivariable logistic regression models for post-TAVR myocardial injury higher 30-day (HR 1.69, 95\% CI 0.38-7.50, $p=0.488$ ) and 1-year all-cause mortality rate (HR $1.90,95 \%$ CI 0.81-4.47, $p=0.139$ ) (Table 7 and Fig. 2).

Cox regression models adjusted for age and sex showed only EUROSCORE II (HR 1.16, 95\% CI 1.08-1.19, $p<0.001)$ and life-threatening/major bleeding complications (HR 5.87, 95\% CI 1.28-26.58, $p=0.019$ ) as independent predictors of 30-day all-cause mortality, and EUROSCORE II (HR 1.08, 95\% CI 1.04-1.13, $p=0.031)$ and AKI (HR $2.59,95 \%$ CI $1.20-5.35, p=0.020$ ) as independent predictors of 1-year all-cause mortality (Table 7).

Finally, similar results consistent to previous Cox models were observed when we repeated Cox regression analysis excluding those patients who received transapical access (Table 8).

\section{Discussion}

In this single-center, retrospective study we found that: (1) PPMI occurs in 2/3rds of patients undergoing TAVR; (2) SEV implantation is associated with a twofold higher incidence of PPMI rate as compared to BEV, even after adjusting for several a priori confounders and procedural variables; (3) PPMI does not significant impact on all-cause 30-day and 1-year mortality. 
Table 5 Univariate and multivariable logistic regression models for post-TAVR myocardial injury adjusted for pre-dilatation, post-dilatation, and rapid pacing

\begin{tabular}{|c|c|c|c|c|c|c|}
\hline \multirow[t]{2}{*}{ Predictor } & \multicolumn{3}{|c|}{ Univariate } & \multicolumn{3}{|c|}{ Multivariate } \\
\hline & OR & $95 \% \mathrm{CI}$ & $p$ value & OR & $95 \% \mathrm{CI}$ & $p$ value \\
\hline Sex (male) & 0.56 & $0.37-0.82$ & 0.390 & 0.76 & $0.46-1.26$ & 0.283 \\
\hline Age & 1.01 & $0.98-1.05$ & 0.466 & 0.97 & $0.92-1.01$ & 0.156 \\
\hline SEV & 2.52 & $1.62-4.05$ & $<0.001$ & 2.99 & $1.59-5.99$ & 0.001 \\
\hline Pre-TAVR CrCl & 0.99 & $0.98-1.00$ & 0.031 & 0.98 & $0.97-1.00$ & 0.015 \\
\hline LVEF & 1.03 & $1.01-1.05$ & $<0.001$ & 1.05 & $1.02-1.07$ & $<0.001$ \\
\hline Diabetes & 0.57 & $0.38-0.87$ & 0.009 & 0.74 & $0.45-1.24$ & 0.244 \\
\hline CAD & 0.58 & $0.39-0.86$ & 0.007 & 0.76 & $0.46-1.25$ & 0.280 \\
\hline Maximum aortic gradient & 1.01 & $1.00-1.02$ & 0.014 & 1.01 & $1.00-1.02$ & 0.178 \\
\hline Pre-dilatation & 1.24 & $0.82-1.86$ & 0.312 & 1.04 & $0.63-1.73$ & 0.867 \\
\hline Post-dilatation & 1.09 & $0.56-2.28$ & 0.809 & 0.87 & $0.41-1.99$ & 0.729 \\
\hline Rapid pacing & 0.65 & $0.34-1.15$ & 0.158 & 1.37 & $0.58-3.24$ & 0.469 \\
\hline
\end{tabular}

$C A D$ coronary artery disease, $\mathrm{CrCl}$ creatinine clearance, $L V E F$ left ventricular ejection fraction, $S E V$ selfexpandable valve, TAVR transcatheter aortic valve replacement

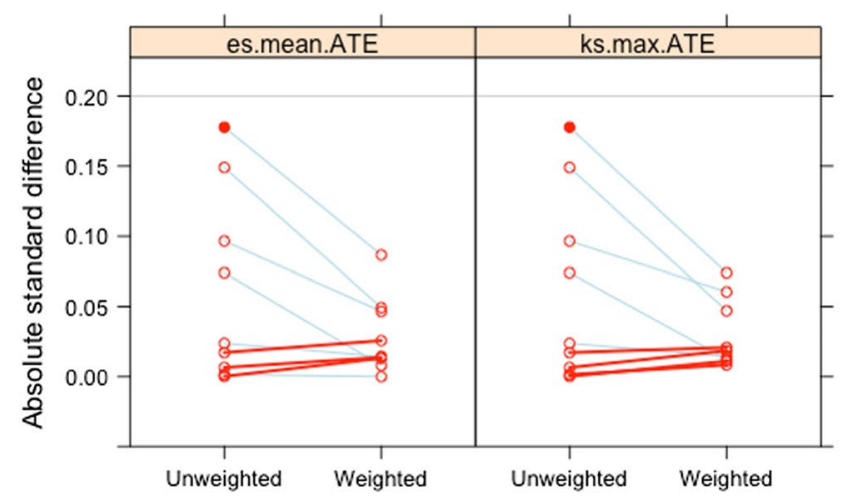

Fig. 1 Results of balancing SEV vs. BEV with an average treatment effect propensity score. a Absolute standard differences for baseline covariates before and after propensity score showing well balancing of covariates: absolute standard differences for covariates decrease between the two investigational groups (BEV vs. SEV) when pro-

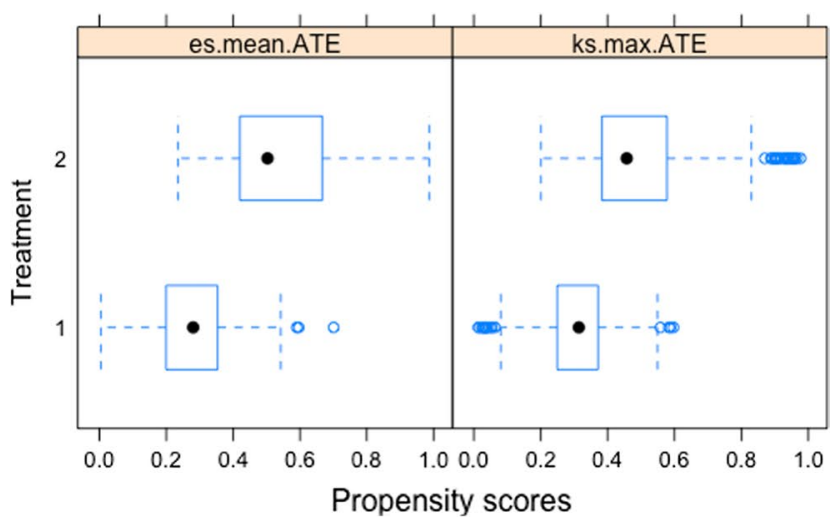

pensity score weighting was applied; b propensity scores for covariates before and after weighting: after well balancing of covariates, the scores appear more overlapping between the two investigational groups (BEV vs. SEV) with less dispersion at extreme values. $B E V$ balloon-expandable valve, $S E V$ self-expandable valve
Table 6 Univariate and multivariable logistic regression models for postTAVR myocardial injury excluding patients who received transapical access

\begin{tabular}{|c|c|c|c|c|c|c|}
\hline \multirow[t]{2}{*}{ Predictor } & \multicolumn{3}{|c|}{ Univariate } & \multicolumn{3}{|c|}{ Multivariate } \\
\hline & OR & $95 \% \mathrm{CI}$ & $p$ value & OR & $95 \% \mathrm{CI}$ & $p$ value \\
\hline Sex (male) & 0.56 & $0.38-0.84$ & 0.005 & 0.71 & $0.42-1.18$ & 0.185 \\
\hline Age & 1.01 & $0.97-1.05$ & 0.599 & 0.97 & $0.92-1.02$ & 0.276 \\
\hline SEV & 2.64 & $1.68-4.24$ & $<0.001$ & 3.48 & $2.01-6.26$ & $<0.001$ \\
\hline Pre-TAVR CrCl & 0.99 & $0.98-1.00$ & 0.031 & 0.98 & $0.97-1.00$ & 0.021 \\
\hline LVEF & 1.04 & $1.02-1.06$ & $<0.001$ & 1.04 & $1.02-1.07$ & $<0.001$ \\
\hline Diabetes & 0.53 & $0.35-0.81$ & 0.003 & 0.66 & $0.40-1.10$ & 0.105 \\
\hline CAD & 0.56 & $0.37-0.84$ & 0.005 & 0.61 & $0.37-0.99$ & 0.056 \\
\hline Maximum aortic gradient & 1.01 & $1.00-1.02$ & 0.012 & 1.01 & $1.00-1.02$ & 0.185 \\
\hline
\end{tabular}

$C A D$ coronary artery disease, $C r C l$ creatinine clearance, $L V E F$ left ventricular ejection fraction, $S E V$ selfexpandable valve, TAVR transcatheter aortic valve replacement 
Table 7 Cox regression models for 30-day and 1-year all-cause mortality

\begin{tabular}{|c|c|c|c|c|c|c|}
\hline & \multicolumn{3}{|c|}{ Univariate } & \multicolumn{3}{|c|}{ Multivariate } \\
\hline & HR & $95 \% \mathrm{CI}$ & $p$ value & $\mathrm{HR}$ & $95 \% \mathrm{CI}$ & $p$ value \\
\hline \multicolumn{7}{|l|}{ 30-day all-cause mortality } \\
\hline EUROSCORE II & 1.15 & $1.06-1.23$ & $<0.001$ & 1.16 & $1.08-1.19$ & $<0.001$ \\
\hline AKI & 1.96 & $1.69-5.49$ & 0.025 & 1.41 & $0.44-4.14$ & 0.548 \\
\hline Vascular complications & 2.86 & $0.98-8.36$ & 0.052 & 1.06 & $0.24-4.31$ & 0.876 \\
\hline Life-threatening/major bleeding complications & 7.64 & $2.61-22.35$ & $<0.001$ & 5.87 & $1.28-26.58$ & 0.019 \\
\hline PPMI & 1.69 & $0.38-7.50$ & 0.488 & - & - & - \\
\hline \multicolumn{7}{|l|}{ 1-year all-cause mortality } \\
\hline EUROSCORE II & 1.09 & $1.03-1.15$ & 0.002 & 1.08 & $1.04-1.13$ & 0.031 \\
\hline AKI & 2.83 & $1.59-5.07$ & $<0.001$ & 2.59 & $1.20-5.35$ & 0.020 \\
\hline Baseline $\mathrm{CrCl}$ & 0.97 & $0.95-0.99$ & 0.002 & 0.97 & $0.96-1.01$ & 0.092 \\
\hline Baseline NT-proBNP & 1.01 & $1.01-1.02$ & 0.003 & 1.00 & $0.99-1.01$ & 0.540 \\
\hline Non-transfemoral access & 1.77 & $1.20-3.95$ & 0.016 & 1.29 & $0.45-3.01$ & 0.510 \\
\hline Life-threatening/major bleeding complications & 4.49 & $2.17-9.27$ & $<0.001$ & 3.13 & $0.81-14.11$ & 0.114 \\
\hline PPMI & 1.90 & $0.81-4.47$ & 0.139 & - & - & - \\
\hline
\end{tabular}

$A K I$ acute kidney injury, $B N P$ brain natriuretic peptide, $C r C l$ creatinine clearance, $P P M I$ post-procedure myocardial injury

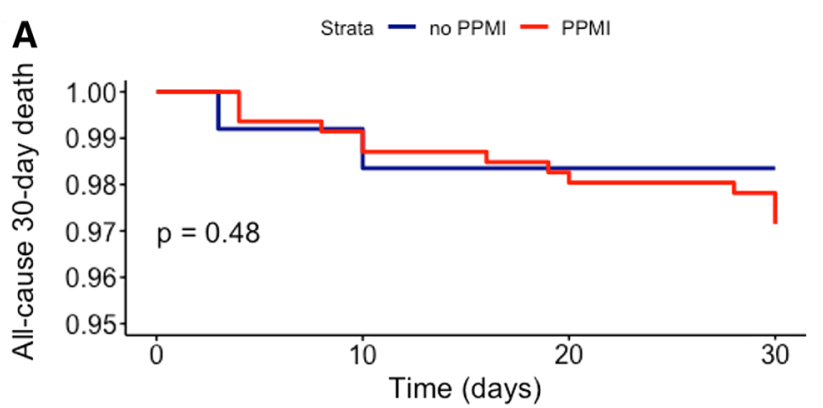

Number at risk

$\begin{array}{rrrrr}\text { no PPMI } & 125 & 117 & 115 & 115 \\ \text { PPMI } & 471 & 453 & 443 & 439\end{array}$

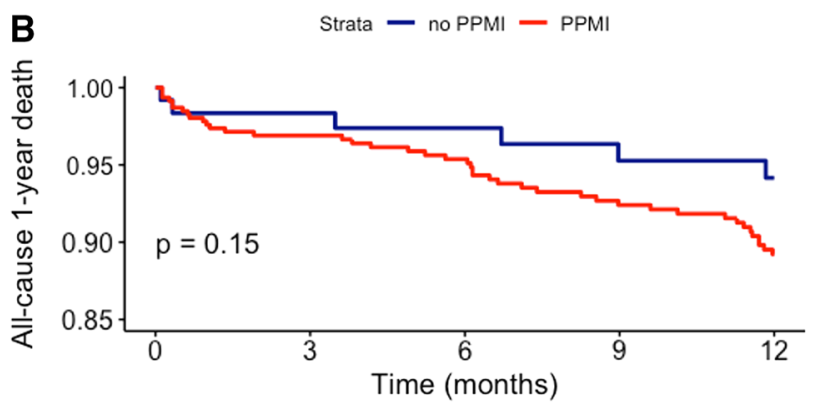

Number at risk

$\begin{array}{rrrrcc}\text { no PPMI } & 125 & 102 & 95 & 88 & 84 \\ \text { PPMI } & 471 & 392 & 365 & 329 & 300\end{array}$

Fig. 2 Kaplan-Meier curves for 30-day (panel A) and 1-year (panel B) all-cause mortality according to PPMI. PPMI post-procedure myocardial injury
Myocardial infarction (MI) is a rare and potentially life-threatening complication of TAVR, usually caused by mechanical interference of prosthesis with coronary ostia, nonetheless a PPMI which is based on bio-humoral markers of myocardial injury is commonly reported and generally clinically silent $[7-9,14-16,19,21]$. In our cohort, we observed a PPMI rate of $79.0 \%$ which is one the highest reported in the literature so far [6, 15, 22-24]. This result is probably explained by several factors: first and foremost, we adopted the new VARC-2 definition of myocardial injury and used the troponin I as the biomarker of choice: TnI seems to be less stable and more related to peri-procedural complications and non-cardiac cause of PPMI (e.g., ischemia related to anemia or dehydration, acute kidney injury, or severe hypoxia due to acute respiratory failure) than CK-MB [19], which was used in some previous studies [7-9]. Second, several baseline characteristics (e.g., lower average $\mathrm{CrCl}$ and higher NYHA class), procedural variables (such as longer procedural times) and incidence of post-procedural complications (e.g., higher significant bleedings or need for pacemaker implantation) resulted higher in ours than in other comparable cohorts, perhaps due to the inclusion of "real-world" patients across 10 years, spanning the learning curve of the center.

In agreement with previous data, we demonstrate an association between PPMI and higher LVEF, probably because viable myocardial tissue results in higher enzymes release, and also with worse baseline kidney function [8, 22-24], whereas we could not observe an independent association between PPMI and pre-TAVR valvuloplasty, mean procedural duration, and previous $\mathrm{MI}$ or CAD as 
Table 8 Cox regression models for 30-day and 1-year all-cause mortality excluding patients who received transapical access

\begin{tabular}{|c|c|c|c|c|c|c|}
\hline & \multicolumn{3}{|c|}{ Univariate } & \multicolumn{3}{|c|}{ Multivariate } \\
\hline & HR & $95 \% \mathrm{CI}$ & $p$ value & HR & $95 \% \mathrm{CI}$ & $p$ value \\
\hline \multicolumn{7}{|l|}{ 30-day all-cause mortality } \\
\hline EUROSCORE II & 1.15 & $1.07-1.24$ & $<0.001$ & 1.13 & $1.05-1.21$ & 0.002 \\
\hline AKI & 2.17 & $1.75-6.25$ & 0.022 & 1.44 & $0.48-4.36$ & 0.514 \\
\hline Vascular complications & 3.11 & $1.04-9.28$ & 0.042 & 1.06 & $0.24-4.66$ & 0.938 \\
\hline Life-threatening/major bleeding complications & 8.47 & $2.84-25.28$ & $<0.001$ & 6.20 & $1.37-28.06$ & 0.018 \\
\hline PPMI & 3.47 & $0.45-26.53$ & 0.230 & - & - & - \\
\hline \multicolumn{7}{|l|}{ 1-year all-cause mortality } \\
\hline EUROSCORE II & 1.08 & $1.02-1.15$ & 0.005 & 1.07 & $1.02-1.15$ & 0.034 \\
\hline AKI & 2.29 & $1.27-4.15$ & 0.006 & 1.27 & $1.13-3.06$ & 0.020 \\
\hline Baseline $\mathrm{CrCl}$ & 0.97 & $0.95-0.99$ & 0.011 & 0.99 & $0.96-1.02$ & 0.132 \\
\hline Baseline NT-proBNP & 1.01 & $1.01-1.02$ & 0.006 & 1.00 & $0.99-1.01$ & 0.198 \\
\hline Life-threatening/major bleeding complications & 4.97 & $2.39-10.35$ & $<0.001$ & 3.99 & $0.91-11.79$ & 0.069 \\
\hline PPMI & 2.19 & $0.86-5.55$ & 0.098 & - & - & - \\
\hline
\end{tabular}

$A K I$ acute kidney injury, $B N P$ brain natriuretic peptide, $C r C l$ creatinine clearance, $P P M I$ post-procedure myocardial injury reported in other cohorts $[8,9,17,22-24]$, probably due to heterogenous sampling and selection biases.

PPMI has a mainly procedural origin, related to mechanical trauma to the myocardium due to contact with valve struts, potential multiple episodes of hypotension during valve release, and myocardial ischemia due to balloon valvuloplasty and/or valve implantation itself $[15,17]$. Of note, we found that SEV implantation might be associated to a higher risk of PPMI has already been reported, but mainly as a collateral result, and without adjustment for other procedural and clinical variables [8, 17, 22-24]. We employed a rigorous analytic approach that included multivariable and non-parsimonious propensity-weighted analysis: the most important implication of our study, thus, is that, when the choice of implanting a SEV is made, a two- to threefold higher risk of PPMI should be accounted.

The reasons for the increased PPMI risk and SEV technology are multiple: whereas in SEV implantation, the need for rapid pacing and the consequent extreme periods of hypotension are less than in BEV, SEV usually leads to peri-valvular myocardial compression (often for greater valve oversizing leading to deeper positioning of the metal frame), the release time can be longer, and there is more arrhythmic potential due to more frequent need for inotropic support $[9,10,15,23,25]$. All these mechanisms may lead to hypoperfusion-induced ischemia and hemodynamic instability occurring more frequently than in BEV. In addition, it could be postulated that BEV require only a brief highpressure balloon inflation during implantation, whereas the self-expanding frame in SEV applies continuous pressure to the surrounding structures, which in turn might result in substantially greater myocardial damage, finally SEV may need multiple repositioning as compared with BEV.
We could not identify a significant relationship between PPMI and 30-day and 1-year all-cause mortality post-TAVR: the literature data are highly controversial on this topic, since several publications $[7,8,17,22,26]$ found that PPMI is clearly linked to higher mortality, whereas other reports have been unable to find any significant association [5, 23, 24]. Nevertheless, a possible reason for this discrepancy is that we evaluated all-cause mortality, whereas PPMI was shown to be mainly associated with cardiovascular mortality [7, $8,22,26]$.

A recent meta-analysis by Michail et al. reported higher overall 30-day (5.2\%) and 1-year (18.6\%) all-cause mortality than our study (2.5\% and $8.1 \%$, respectively) [17]. Furthermore, most previous publications included TAVR cohorts with a significant prevalence of femoral surgical and transapical access and with substantial rates of complications such as pericardiocentesis, pericardiotomy, and conversion to open heart surgery [5, 7-9, 14, 17, 19, 21]. Since we limited our analysis to successful TAVR and there was a very low rate of serious complications in the present study, as well as only 15/596 transapical procedures, PPMI conceivably did not affect the prognosis as its association with adverse events affecting mortality was blunted.

In conclusion, the debate on the prognostic role of PPMI after TAVR and its variable link with poor outcomes is still open similarly to that of type 4A MI after coronary interventions [27-31]; in this scenario, our data support the concept that PPMI occurs quite commonly and may mainly represent a "proxy" of higher comorbidity or of peri-procedural complications.

PPMI risk is two- to threefold higher when SEV are used as compared with BEV, but it does not impact all-cause mortality. Therefore, dedicated studies with careful patients' 
selection and a prospective design are needed to define the role of valve different technologies on PPMI.

\section{Limitations}

First, we did not measure CK-MB, which is more stable than TnI and less prone to non-cardiac rise due to several acute and chronic comorbidities [19]. Second, despite we recorded the history of prior $\mathrm{CAD}$, we had no information on the completeness of revascularization pre-TAVR. However, incomplete revascularization seems to not affect PPMI in previous studies $[5,6]$. In addition, we have no data on aortic annulus manipulation (i.e., repositioning/retrieval of the valve) and we did not evaluate myocardial injury with more accurate imaging technique (e.g., cardiac magnetic resonance). Finally, patients were not randomly allocated to a specific valve type, albeit propensity-weighted analysis on the average treatment effect could reduce this bias.

\section{Acknowledgements None.}

Author contributions All the authors contributed to the study conception and design. Material preparation, data collection and analysis were performed by VDM, SB, FP, and MS. The first draft of the manuscript was written by VDM, and all the authors commented on previous versions of the manuscript. All the authors read and approved the final manuscript.

Funding Open access funding provided by Università degli Studi di Genova within the CRUI-CARE Agreement.

\section{Declarations}

\section{Conflict of interest None.}

Open Access This article is licensed under a Creative Commons Attribution 4.0 International License, which permits use, sharing, adaptation, distribution and reproduction in any medium or format, as long as you give appropriate credit to the original author(s) and the source, provide a link to the Creative Commons licence, and indicate if changes were made. The images or other third party material in this article are included in the article's Creative Commons licence, unless indicated otherwise in a credit line to the material. If material is not included in the article's Creative Commons licence and your intended use is not permitted by statutory regulation or exceeds the permitted use, you will need to obtain permission directly from the copyright holder. To view a copy of this licence, visit http://creativecommons.org/licenses/by/4.0/.

\section{References}

1. Genereux P, Head SJ, Van Mieghem NM, Kodali S, Kirtane AJ, Xu K, Smith C, Serruys PW, Kappetein AP, Leon MB (2012) Clinical outcomes after transcatheter aortic valve replacement using valve academic research consortium definitions: a weighted meta-analysis of 3,519 patients from 16 studies. J Am Coll Cardiol 59(25):2317-2326
2. Leon MB, Smith CR, Mack M, Miller DC, Moses JW, Svensson LG, Tuzcu EM, Webb JG, Fontana GP, Makkar RR, Brown DL, Block PC, Guyton RA, Pichard AD, Bavaria JE, Herrmann HC, Douglas PS, Petersen JL, Akin JJ, Anderson WN, Wang D, Pocock S, Investigators PT (2010) Transcatheter aortic-valve implantation for aortic stenosis in patients who cannot undergo surgery. N Engl J Med 363(17):1597-1607

3. Smith CR, Leon MB, Mack MJ, Miller DC, Moses JW, Svensson LG, Tuzcu EM, Webb JG, Fontana GP, Makkar RR, Williams M, Dewey T, Kapadia S, Babaliaros V, Thourani VH, Corso P, Pichard AD, Bavaria JE, Herrmann HC, Akin JJ, Anderson WN, Wang D, Pocock SJ, Investigators PT (2011) Transcatheter versus surgical aortic-valve replacement in high-risk patients. N Engl J Med 364(23):2187-2198

4. Vahanian A, Alfieri O, Al-Attar N, Antunes M, Bax J, Cormier B, Cribier A, De Jaegere P, Fournial G, Kappetein AP, Kovac J, Ludgate S, Maisano F, Moat N, Mohr F, Nataf P, Pierard L, Pomar JL, Schofer J, Tornos P, Tuzcu M, van Hout B, Von Segesser LK, Walther T (2008) Transcatheter valve implantation for patients with aortic stenosis: a position statement from the European association of cardio-thoracic surgery (EACTS) and the European Society of Cardiology (ESC), in collaboration with the European Association of Percutaneous Cardiovascular Interventions (EAPCI). EuroIntervention 4(2):193-199

5. Kim WK, Liebetrau C, van Linden A, Blumenstein J, Gaede L, Hamm CW, Walther T, Mollmann H (2016) Myocardial injury associated with transcatheter aortic valve implantation (TAVI). Clin Res Cardiol 105(5):379-387

6. Koskinas KC, Stortecky S, Franzone A, O'Sullivan CJ, Praz F, Zuk K, Raber L, Pilgrim T, Moschovitis A, Fiedler GM, Juni P, Heg D, Wenaweser P, Windecker S (2016) Post-Procedural troponin elevation and clinical outcomes following transcatheter aortic valve implantation. J Am Heart Assoc 5(2):e002430

7. Ribeiro HB, Nombela-Franco L, Munoz-Garcia AJ, Lemos PA, Amat-Santos I, Serra V, Brito FS, Abizaid A, Sarmento-Leite R, Puri R, Cheema AN, Ruel M, Nietlispach F, Maisano F, Moris C, Del Valle R, Urena M, Abdul Jawad Altisent O, Del Trigo M, Campelo-Parada F, Jimenez Quevedo P, Alonso-Briales JH, Gutierrez H, Garcia Del Blanco B, Perin MA, Siqueira D, Bernardi G, Dumont E, Cote M, Pibarot P, Rodes-Cabau J (2015) Predictors and impact of myocardial injury after transcatheter aortic valve replacement: a multicenter registry. J Am Coll Cardiol 66(19):2075-2088

8. Rodes-Cabau J, Gutierrez M, Bagur R, De Larochelliere R, Doyle D, Cote M, Villeneuve J, Bertrand OF, Larose E, Manazzoni J, Pibarot P, Dumont E (2011) Incidence, predictive factors, and prognostic value of myocardial injury following uncomplicated transcatheter aortic valve implantation. J Am Coll Cardiol 57(20):1988-1999

9. Yong ZY, Wiegerinck EM, Boerlage-van Dijk K, Koch KT, Vis MM, Bouma BJ, Henriques JP, Cocchieri R, Piek JJ, de Mol BA, Baan J Jr (2012) Predictors and prognostic value of myocardial injury during transcatheter aortic valve implantation. Circ Cardiovasc Interv 5(3):415-423

10. Kahlert P, Al-Rashid F, Plicht B, Wild C, Westholter D, Hildebrandt H, Baars T, Neumann T, Nensa F, Nassenstein K, Wendt D, Thielmann M, Jakob H, Kottenberg E, Peters J, Erbel R, Heusch G (2016) Myocardial injury during transfemoral transcatheter aortic valve implantation: an intracoronary Doppler and cardiac magnetic resonance imaging study. EuroIntervention 11(12):1401-1408

11. Kim WK, Rolf A, Liebetrau C, Van Linden A, Blumenstein J, Kempfert J, Bachmann G, Nef H, Hamm C, Walther T, Mollmann H (2014) Detection of myocardial injury by CMR after transcatheter aortic valve replacement. J Am Coll Cardiol 64(4):349-357 
12. Nilsson L, Appel CF, Hultkvist H, Vanky F (2015) Evaluation of the valve academic research consortium- 2 criteria for myocardial infarction in transcatheter aortic valve implantation: a prospective observational study. PLoS ONE 10(6):e0130423

13. Saia F, Marrozzini C, Marzocchi A (2011) Displacement of calcium nodules of the native valve as a possible cause of left main occlusion following transcatheter aortic valve implantation. J Invasive Cardiol 23(5):E106-109

14. Barbash IM, Dvir D, Ben-Dor I, Badr S, Okubagzi P, Torguson R, Corso PJ, Xue Z, Satler LF, Pichard AD, Waksman R (2013) Prevalence and effect of myocardial injury after transcatheter aortic valve replacement. Am J Cardiol 111(9):1337-1343

15. Sinning JM, Hammerstingl C, Schueler R, Neugebauer A, Keul S, Ghanem A, Mellert F, Schiller W, Muller C, Vasa-Nicotera M, Zur B, Welz A, Grube E, Nickenig G, Werner N (2016) The prognostic value of acute and chronic troponin elevation after transcatheter aortic valve implantation. EuroIntervention 11(13):1522-1529

16. Leon MB, Smith CR, Mack MJ, Makkar RR, Svensson LG, Kodali SK, Thourani VH, Tuzcu EM, Miller DC, Herrmann HC, Doshi D, Cohen DJ, Pichard AD, Kapadia S, Dewey T, Babaliaros V, Szeto WY, Williams MR, Kereiakes D, Zajarias A, Greason KL, Whisenant BK, Hodson RW, Moses JW, Trento A, Brown DL, Fearon WF, Pibarot P, Hahn RT, Jaber WA, Anderson WN, Alu MC, Webb JG, Investigators P (2016) Transcatheter or surgical aortic-valve replacement in intermediate-risk patients. $\mathrm{N}$ Engl J Med 374(17):1609-1620

17. Michail M, Cameron JN, Nerlekar N, Ihdayhid AR, McCormick LM, Gooley R, Niccoli G, Crea F, Montone RA, Brown AJ (2018) Periprocedural myocardial injury predicts short- and long-term mortality in patients undergoing transcatheter aortic valve replacement. Circ Cardiovasc Interv 11(11):e007106

18. Apple FS, Sandoval Y, Jaffe AS, Ordonez-Llanos J, Bio-Markers ITFoCAoC (2017) Cardiac troponin assays: guide to understanding analytical characteristics and their impact on clinical care. Clin Chem 63(1):73-81

19. Kappetein AP, Head SJ, Genereux P, Piazza N, van Mieghem NM, Blackstone EH, Brott TG, Cohen DJ, Cutlip DE, van Es GA, Hahn RT, Kirtane AJ, Krucoff MW, Kodali S, Mack MJ, Mehran R, Rodes-Cabau J, Vranckx P, Webb JG, Windecker S, Serruys PW, Leon MB, Valve Academic Research Consortium (2012) Updated standardized endpoint definitions for transcatheter aortic valve implantation: the Valve Academic Research Consortium-2 consensus document. EuroIntervention 8(7):782-795

20. McCaffrey DF, Griffin BA, Almirall D, Slaughter ME, Ramchand R, Burgette LF (2013) A tutorial on propensity score estimation for multiple treatments using generalized boosted models. Stat Med 32(19):3388-3414

21. Paradis JM, Maniar HS, Lasala JM, Kodali S, Williams M, Lindman BR, Damiano RJ Jr, Moon MR, Makkar RR, Thourani VH, Babaliaros V, Xu K, Ayele GM, Svensson L, Leon MB, Zajarias A (2015) Clinical and functional outcomes associated with myocardial injury after transfemoral and transapical transcatheter aortic valve replacement: a subanalysis from the partner trial (placement of aortic transcatheter valves). JACC Cardiovasc Interv 8(11):1468-1479

22. Nara Y, Watanabe Y, Kataoka A, Nakashima M, Hioki H, Nagura F, Kawashima H, Konno K, Kyono H, Yokoyama N, Kozuma K
(2018) Incidence, predictors, and midterm clinical outcomes of myocardial injury after transcatheter aortic-valve implantation. Int Heart J 59(6):1296-1302

23. Rahhab Z, Labarre Q, Nijenhuis VJ, El Faquir N, de Biase C, Philippart R, Heijmen R, Kardys I, Dumonteil N, de Jaegere P, van der Heijden J, Tchetche D, Van Mieghem NM (2019) Myocardial injury post transcatheter aortic valve implantation comparing mechanically expanded versus self-expandable versus balloonexpandable valves. Struct Heart 3(5):431-437

24. Stundl A, Schulte R, Lucht H, Weber M, Sedaghat A, Shamekhi J, Zur B, Grube E, Mellert F, Welz A, Fimmers R, Nickenig G, Werner N, Sinning JM (2017) Periprocedural myocardial injury depends on transcatheter heart valve type but does not predict mortality in patients after transcatheter aortic valve replacement. JACC Cardiovasc Interv 10(15):1550-1560

25. Weber M, Bazzino O, Navarro Estrada JL, de Miguel R, Salzberg S, Fuselli JJ, Liebetrau C, Woelken M, Moellmann H, Nef H, Hamm C (2011) Improved diagnostic and prognostic performance of a new high-sensitive troponin $\mathrm{T}$ assay in patients with acute coronary syndrome. Am Heart J 162(1):81-88

26. Sharma V, Dey T, Sankaramangalam K, Alansari SAR, Williams L, Mick S, Krishnaswamy A, Svensson LG, Kapadia S (2019) Prognostically significant myocardial injury in patients undergoing transcatheter aortic valve replacement. J Am Heart Assoc 8(14): 011889

27. Liou K, Jepson N, Kellar P, Ng B, Isbister J, Giles R, Friedman D, Allan R, Lau A, Pitney M, Ooi SY (2015) Prognostic significance of peri-procedural myocardial infarction in the era of high sensitivity troponin: a validation of the joint ACCF/AHA/ESC/ WHF universal definition of type 4a myocardial infarction with high sensitivity troponin T. Heart Lung Circ 24(7):673-681

28. Lozano I, Rondan J, Vegas JM, Segovia E (2019) Definition of myocardial infarction type 4a: can we define its diagnosis and systematize clinical practice? Rev Esp Cardiol (Engl Ed) 72(8):695-696

29. Moussa ID, Klein LW, Shah B, Mehran R, Mack MJ, Brilakis ES, Reilly JP, Zoghbi G, Holper E, Stone GW (2013) Consideration of a new definition of clinically relevant myocardial infarction after coronary revascularization: an expert consensus document from the Society for Cardiovascular Angiography and Interventions (SCAI). J Am Coll Cardiol 62(17):1563-1570

30. Yang X, Tamez H, Lai C, Ho K, Cutlip D (2017) Type 4a myocardial infarction: Incidence, risk factors, and long-term outcomes. Catheter Cardiovasc Interv 89(5):849-856

31. Zeitouni M, Silvain J, Guedeney P, Kerneis M, Yan Y, Overtchouk P, Barthelemy O, Hauguel-Moreau M, Choussat R, Helft G, Le Feuvre C, Collet JP, Montalescot G, Group AS (2018) Periprocedural myocardial infarction and injury in elective coronary stenting. Eur Heart J 39(13):1100-1109

Publisher's Note Springer Nature remains neutral with regard to jurisdictional claims in published maps and institutional affiliations. 